\title{
Contribuições do Grupo Terapêutico Educação em Saúde na motivação para a vida do usuário de substâncias psicoativas
}

Recebido em: 11/04/2012

Aceito em: 16/04/2012

\author{
Selene Cordeiro Vasconcelos \\ Iracema da Silva Frazão \\ Vânia Pinheiro Ramos ${ }^{3}$
}

\begin{abstract}
Objetivo: Compreender as contribuições do Grupo Terapêutico Educação em Saúde na motivação para a vida de usuários de substâncias psicoativas. Metodologia: Estudo descritivo, exploratório com abordagem qualitativa, envolvendo oito usuários de um CAPSad. Foram realizadas consultas em prontuário e entrevistas submetidas à análise de conteúdo. Resultados: as contribuições foram identificadas como uma força a mais, um nascer de novo, um aprendizado ao enfrentamento das dificuldades. Conclusão: esse Grupo tem proporcionado um ambiente de troca de saberes entre os participantes e de suporte para aquisição de hábitos saudáveis.
\end{abstract}

Descritores: Enfermagem, Educação em Saúde, Autocuidado, Transtornos Relacionados ao Uso de Substâncias.

\section{Health Education therapy group contributions in the motivation to the psychoactive substances user's life}

Objective: To understand the contributions of Group Therapy Health Education in motivation to the lives of drug users. Methodology: A descriptive, exploratory qualitative approach, involving users in eight CAPSad. Consultations were held in medical records and interviews were submitted to content analysis. Results: contributions have been identified as a strength the most, a born again, a learning to cope with the difficulties. Conclusion: This group has provided an environment for knowledge exchange between participants and support for the acquisition of healthy habits.

Descriptors: Nursing, Health Education, Self Care, Disorders Related to Substance Use.

\section{Contribuciones del grupo terapéutico educación en salud en la motivación para la vida del usuario de substancias psicoactivas}

Objetivo: Comprender la contribución del grupo terapéutico educación en Salud en la motivación para la vida de los usuarios de drogas. Metodología: Un enfoque exploratorio descriptivo, cualitativo, con la participación de usuarios en ocho CAPSad. Se celebraron consultas en los registros médicos y entrevistas fueron sometidas a análisis de contenido. Resultados: Contribuciones han sido identificados como una fuerza más, los nacidos de nuevo, el aprendizaje para hacer frente a las dificultades. Conclusión: Este grupo ha creado un entorno para el intercambio de conocimientos entre los participantes y el apoyo para la adquisición de hábitos saludables.

Descriptores: Enfermería, Educación Sanitaria, Cuidados Personales, Los Trastornos Relacionados con el Consumo de Sustancias

\section{INTRODUÇÃO}

$\mathrm{O}$ cuidar é uma prática constante na vida das pessoas, uma necessidade para a sobrevivência. Ele pode ser recebido antes mesmo do nascimento e acompanha todo o desenvolvimento humano num contínuo aprendizado, tornando-o capaz de cuidar do outro e de exercer o seu autocuidado, o que ajuda a manter a sua integridade estrutural e funcional. A prática do autocuidado pode ser conceituada, dessa forma, como o desempenho ou a realização de atividades que as pessoas exercem em seu benefício para manter a vida, a saúde e o bem-estar ${ }^{(1)}$.

Assim como o cuidar, o uso de drogas é uma prática que também tem acompanhado a história da humanidade, porém sob diferentes formas, como chás, fumos mágicos e óleos medicinais, todas controladas por normas sociais e ritos, tendo tanto função ritualística ou mística e curativa quanto busca do prazer $^{(2)}$. No entanto, essa prática necessita de um cuidar especializado, que compre às subseenda a complexidade do fenômeno "uso de drogas" e a dinâmica do usuário.

O termo "drogas" refere-stâncias psicoativas, que são aquelas que alteram os sentidos ou o psiquismo(3), podendo ou não causar dependência. Como principais consequências tem-se sofrimentos físicos, psíquicos e morais dos usuários e das famílias, preocupando a sociedade e principalmente os profissionais de saúde mental, que têm buscado soluções de forma ampla e intersetorial.

O alcoolismo é considerado pela Organização Mundial

1 Enfermeira. Mestre em Enfermagem. Enfermeira assistencial do CAPSad Eulâmpio Cordeiro, Recife-PE e do Serviço de Pronto Atendimento - CEMEC, Camaragibe-PE. E-mail: selumares@yahoo.com.br

2 Enfermeira. Doutora em Serviço Social. Professora Adjunta 3 Universidade Federal de Pernambuco - UFPE.

3 Enfermeira. Doutora em Neurociências. Professora Adjunta 4 Universidade Federal de Pernambuco - UFPE. 
de Saúde (OMS) como um problema de saúde pública por ocasionar para a sociedade uma carga considerável de agravos indesejáveis, como alta mortalidade e incapacidade ${ }^{(4)}$. Outro aspecto é que, no Brasil, o consumo de bebidas alcoólicas tem se iniciado cada vez mais cedo entre os jovens ${ }^{(5)}$, realidade que também ocorre em países desenvolvidos ${ }^{(4)}$. Outro agravante é o número crescente de internações devido ao uso de outras drogas associadas ou não ao uso do álcool, o que tem acometido várias faixas etárias, gerando grande prejuízo à sociedade ${ }^{(6)}$.

Sabe-se que o uso de substâncias psicoativas está relacionado a outros fenômenos como morte prematura, redução de dias de vida produtiva, sendo frequentemente associado à diminuição da motivação para a vida e considerado como comportamento destrutivo e de morte.

Os prejuízos físicos, sociais e psicológicos decorrentes do uso de drogas comprometem a produtividade e o desempenho profissional do usuário ${ }^{(7)}$. Salienta-se que os anos produtivos de vida perdidos por morte precoce atingem não só a pessoa, mas também todas as suas relações econômicas e intelectuais com a coletividade ${ }^{(8)}$.

Nesse contexto, percebe-se a necessidade de um cuidado especializado para esses usuários, trabalho que vem sendo desenvolvido em Centros de Atenção Psicossocial para Álcool e outras Drogas (CAPSad), órgão que, no âmbito da cidade do Recife, é composto por unidades de saúde vinculadas ao Projeto Mais Vida, da Secretaria de Saúde desse município, tendo a Política de Redução de Danos como eixo teórico.

Esse cuidado é exercido por uma equipe multiprofissional e principalmente por meio de grupos nos quais os usuários têm a oportunidade de refletir, compartilhar experiências e saberes. Em geral, funcionam como grupos operativos em que o coordenador deve manter-se centrado na tarefa proposta, que pode ser reflexão, manejo de situações conflituosas, estímulo cognitivo, dentre outras ${ }^{(9)}$.

No intuito de analisar as práticas de cuidado aos usuários de substâncias psicoativas, optou-se por estudar a influência do Grupo Terapêutico Educação em Saúde na motivação para a vida.

Portanto, a presente pesquisa tem como objetivo compreender as contribuições do Grupo Terapêutico Educação em Saúde na motivação para a vida do usuário de substâncias psicoativas.

\section{METODOLOGIA}

Trata-se de um estudo descritivo e exploratório com abordagem qualitativa, realizado no Centro de Atenção Psicossocial para Álcool e outras Drogas (CAPSad) Eulâmpio Cordeiro, referência para o tratamento de usuários de substâncias psicoativas do Distrito Sanitário IV da Cidade do Recife-PE.

Nesse CAPSad é utilizado o Projeto Terapêutico Singular como um conjunto de ações pactuadas entre o usuário de substâncias psicoativas e o profissional responsável pelos atendimentos individuais durante seu tratamento, nomeado Técnico de Referência. A coleta de dados ocorreu durante os meses de julho e agosto de 2011, a amostragem foi intencional e o número de participantes foi determinado por saturação teórica ${ }^{(5)}$, totalizando oito usuários de substâncias psicoativas que participavam regularmente do Grupo Educação em Saúde no turno da tarde, sob a coordenação da pesquisadora.

A escolha desses sujeitos se justificou por acreditar que a existência de um vínculo terapêutico e de uma relação de confiança com a pesquisadora facilita o compartilhar de sentimentos, emoções, vivências e histórias de vida cotidiana.

As sessões do grupo tinham duração de 1 hora, uma vez por semana, sendo abordados diversos assuntos relativos ao processo saúde-doença no uso de substâncias psicoativas.

Todos os sujeitos aceitaram participar do estudo durante o período de coleta de dados, respondendo à entrevista ${ }^{(5)}$ semiestruturada de forma espontânea e consentindo a gravação em áudio. O roteiro de entrevista foi composto por três questões norteadoras. Após a realização das entrevistas, procedeu-se à transcrição na íntegra das mesmas com posterior leitura e releitura exaustiva para realizar a análise de conteúdo de acordo com Bardin ${ }^{(10)}$. O tratamento e interpretação dos dados foram organizados considerando constructos da Teoria do Autocuidado de Dorothea Orem ${ }^{(11)}$.

A investigação respeitou os princípios bioéticos postulados na Resolução 196/96 do Conselho Nacional de Saúde/ Ministério da Saúde ${ }^{(12)}$. Foi aprovado pelo Comitê de Ética em Pesquisa (CEP) da Universidade Federal de Pernambuco, CAAE 0075.0.172.000-11. Para manter o sigilo e preservar a identidade dos usuários utilizaram-se nomes bíblicos para identificá-los: Amós, Moisés, Davi, Jonas, Pedro, Sara, Ester e Rute.

\section{RESULTADOS}

Os resultados estão apresentados em forma de quadro e textos com a finalidade de organizar os dados coletados.

A caracterização dos usuários está descrita no Quadro 1. Considera-se oportuno explicar que foi considerada como droga de impacto aquela substância identificada pelo usuário como sendo a causadora de seus prejuízos e que motivou seu tratamento.

Ressalta-se que dos oito sujeitos de pesquisa, somente dois eram usuários de crack, os demais usavam álcool; destes apenas um possuía diagnóstico de comorbidade psiquiátrica e somente um possuía diagnóstico de uso nocivo, os demais eram dependentes. Quanto à escolaridade, dois não eram alfabetizados e dois possuíam ensino médio completo. Apenas um possuía renda individual e os demais eram sustentados pelas famílias. Três usuários eram solteiros, quatro separados e apenas uma usuária permanecia casada.

Considerou-se uso nocivo como um padrão de uso que causa dano à saúde física ou mental e dependência como um padrão mal adaptativo de uso de substâncias com repercussões psicológicas, físicas e sociais resultantes da interação entre o ser humano e uma substância psicoativa ${ }^{(13)}$.

A motivação para a vida emergiu a partir do discurso dos usuários ao fazerem considerações sobre a importância do Grupo Educação em Saúde como uma força a mais, percebida 


\begin{tabular}{|l|l|l|l|l|l|l|l|l|}
\hline Usuário & $\begin{array}{l}\text { Idade } \\
\text { (anos) }\end{array}$ & $\begin{array}{l}\text { Início do uso } \\
\text { (anos) }\end{array}$ & Escolaridade & $\begin{array}{l}\text { Estado } \\
\text { civil }\end{array}$ & $\begin{array}{l}\text { Renda } \\
\text { pessoal }\end{array}$ & $\begin{array}{l}\text { Com } \\
\text { quem mora }\end{array}$ & $\begin{array}{l}\text { Comorbidade } \\
\text { Associada (CID 10) }\end{array}$ \\
\hline AMÓS & 50 & 16 & EFI & Solteiro & SRF & Mãe e sobrinha & Não & Álcool (F 10.2) \\
\hline MOISÉS & 34 & 18 & EMC & Separado & SRF & Pais & Não & Álcool (F 10.2) \\
\hline DAVI & 49 & 16 & EFI & Separado & SRF & Mãe, duas irmãs & Não & Álcool (F 10.2) \\
\hline JONAS & 30 & 14 & EFI & Solteiro & SRF & Tia, tio e dois primos & Não & Álcool (F 10.2) \\
\hline PEDRO & 50 & 19 & NA & Separado & SRF & Mãe e duas tias & Não & Álcool (F 10.2) \\
\hline SARA & 24 & 23 & EMC & Separada & SRF & Mãe, irmã e sua filha & Não & Crack (F 14.1) \\
\hline ESTER & 46 & 19 & NA & Casada & 1 SM & Esposo, mãe, filha e & F 33 & Álcool (F 10.2) \\
\hline RUTE & 22 & 19 & EMI & Solteira & SRF & Companheira & Não & Crack (F 14.2) \\
\hline
\end{tabular}

Quadro 1 - Caracterização dos sujeitos da pesquisa. Recife-PE, 2011

como uma expressão de amor e liberdade, forma de preservação de sua vida traduzido por sua fala:

[...]vocês me deram uma força a mais[...]eu acho que já estaria morto[...]eu só fazia me destruir[...] ensina a se libertar[...]ensina a se amar, a querer viver mais. (Amós)

A motivação para a vida também foi reconhecida por meio da descoberta pelo usuário de sua capacidade autodestrutiva. Ao reconhecer esse aspecto do próprio comportamento, o usuário pode refletir e construir atitudes diferentes, mais adequadas para o enfrentamento das situações difíceis e se perceber capaz de realizar mudanças na própria vida, o que foi retratado na fala abaixo:

[...]tava precisando de ajuda, eu não tinha enxergado ainda o meu potencial negativo para o lado do vício[...]você aprende[...]a ser humano[...]. (Moisés)

O tema emergiu ainda como uma forma de proteção e enfrentamento das situações externas, demonstrando fortalecimento enquanto pessoa em tratamento como observado:

tô aprendendo a lidar com as coisas lá fora[...] grupo é pra fortalecer[...]andar com um escudo. (Davi)

Esse tema também foi relacionado a recomeço, que, para Pedro, ocorreu a partir de seu reconhecimento sobre sua negligência com a própria vida, como em sua fala:

Eu não tava ligando pra vida, era como eu tivesse esquecido o mundo. Mas agora não, tô achando que eu nasci de novo[...]me deu uma força. (Pedro)

Outra usuária relata o mesmo sentimento, acrescido de mudança e resgate da confiança das pessoas:

Hoje eu posso dizer que nasci de novo, por que as pessoas me respeitam, confiam em mim[...]mudou muito, mudou tudo, na minha vida mudou tudo. (Ester)

Ao identificar os prejuízos decorrentes do uso das drogas, os usuários retratam a motivação da vida como expressão de um aprendizado e competência para identificar aspectos bons e ruins em sua vida, elaborar suas transformações e construir uma perspectiva de vida diferente.

O desejo de retomar a vida, realizar atividades que dão prazer e satisfação, foi trazido como uma forma de motivação para a vida:

[...]é um apoio[...]eu tô bem, tô feliz e não estou mais usando drogas[...] tô conseguindo retomar a minha vida[...] voltei a estudar, tô praticando esportes que é uma coisa que eu gosto. (Rute)

Apesar da problemática oriunda do uso de substâncias psicoativas, que acarreta prejuízos sociais, afetivos, laborais e orgânicos, que afetam a vida em sua complexidade, alterando inclusive a dinâmica familiar, todos os usuários enxergaram e relataram motivação para a vida e desejo de mudança.

\section{DISCUSSÃO}

Segundo Orem, autocuidado é o desenvolvimento de atividades que as pessoas realizam em seu benefício para manter a vida, a saúde e o bem-estar, colaborando para manter a integridade estrutural e o funcionamento humano(11).

Salienta-se que o usuário de substâncias psicoativas apresenta demandas terapêuticas principalmente relacionadas aos problemas decorrentes de seus comportamentos desadaptados(14). Compreender essa realidade auxilia a enfermagem no planejamento de ações educativas específicas, estimular o autocuidado e o enfrentamento das situações adversas.

Por isso, a enfermagem insere-se nos diferentes cenários do cuidar, podendo atuar de diversas formas e abordagens, destacando-se o grupo terapêutico, que proporciona o compartilhamento de projetos que auxiliam na reconstrução da história de cada pessoa ${ }^{(15)}$, sendo também um ambiente propício para a educação em saúde por facilitar a troca de informações, a reflexão sobre os problemas de saúde e a construção de uma visão crítica sobre o estado de saúde dos envolvidos ${ }^{(16)}$. 
Diante do exposto, percebe-se que o Grupo Terapêutico Educação em Saúde proporcionou motivação para a vida por representar um resgate da esperança, um reforço da autonomia e responsabilização pelo próprio processo de cuidar.

Todos relataram mudanças importantes que repercutiram na motivação para a vida, como se amar, querer viver mais (Amós); conhecer-se melhor (Moisés); não usar álcool, não ficar na rua (Davi); nascer de novo (Pedro); ter alegria, ser boa filha e boa mãe (Sara); nascer de novo, mudança de vida (Ester); retomar a vida com um todo, não usar drogas (Rute).

A promoção da saúde relaciona-se com qualidade de vida e engloba ações coletivas, num sentido mais amplo e agregado a políticas públicas, contribuindo para aquisição de estilo de vida responsável e de cuidados de saúde ${ }^{(17)}$. Envolver saberes técnicos e populares contribui para a construção do processo saúde-doença-cuidado e consequentemente para a promoção da saúde e do bem-estar(18).

\section{CONCLUSÕES}

Diante do exposto, conclui-se que o Grupo Terapêutico Educação em Saúde constitui um espaço de cuidado capaz de realizar uma motivação para a vida por meio da troca de saberes, vivências e experiências entre os envolvidos no processo grupal, bem como oportuniza ao enfermeiro perceber a dinâmica do fenômeno do uso de substâncias psicoativas em sua complexidade, contribuindo para seu manejo de grupo.

Reconhecer o autocuidado como um processo contínuo de aprendizado e que depende da escolha voluntária da pessoa em se engajar nele, sofrendo influências sociais e culturais, também auxilia o enfermeiro a planejar e implementar ações educativas mais específicas para essa problemática, respeitando a liberdade de escolha do usuário, estimulando a construção e compartilhamento de saberes.

Torna-se oportuno refletir sobre a dimensão da problemática vivenciada pelo usuário de substâncias psicoativas que abrange prejuízos em diversas áreas de sua vida, principalmente em relação aos vínculos familiares, ao exercício da cidadania e ao convívio social saudável.

Portanto, o ambiente proporcionado pelo Grupo Terapêutico Educação em Saúde tem contribuído para o fortalecimento dos usuários de substâncias psicoativas para o enfrentamento das situações adversas, manifestando-se como uma motivação para a vida, evidenciada pela aquisição de uma postura mais ativa e responsável em seu tratamento, reconhecendo-se como sujeitos de suas histórias de vida.

\section{Referências}

1. Vargas D, Oliveira MAF, Luís MAV. Atendimento ao alcoolista em serviços de atenção primária à saúde: percepções e condutas do enfermeiro. Acta Paul Enferm. 2010;23(1):73-9.

2. Corradi-Webster CM, Esper LH, Pillon SC. A enfermagem e a prevenção do uso indevido de drogas entre adolescentes. Acta Paul Enferm. 2009;22(3):331-4. 3. Laranjeira R, Bordin S, Figlie NB. Aconselhamento em dependência química. São Paulo: Roca; 2010.

4. Oliveira IBS. Tecendo saberes: fenomenologia do tratamento da dependência química [dissertação]. Pará: Universidade Federal do Pará - Instituto de Filosofia e Ciências Humanas; 2007.

5. Flick U. Introdução à pesquisa qualitativa. 3a Ed. Porto Alegre: Artmed; 2009. 6. Araújo MAL, Pagliuca LMF. Análisis de contexto del concepto de ambiente en la Teoria Humanística de Parterson y Zderad. Index Enferm. 2005;16(48-9):42-4. 7. Beck LM, David HMSL. O abuso de drogas e o mundo do trabalho: possibilidades de atuação para o enfermeiro. Esc Anna Nery Rev Enferm. 2007;11(4):706-11.

8. Pereira MSLC, Ferreira LOC, Silva GA, Lucio PS. Evolução da mortalidade e dos anos potenciais e produtivos de vida perdidos por câncer de mama em mulheres no Rio Grande do Norte, entre 1988 e 2007. Epidemiol Serv Saúde. 2011;20(2):161-72.

9. Landim CAP. A competência de pessoas com diabetes mellitus para o autocuidado em um programa educativo multiprofissional [dissertação]. Ribeirão Preto (SP): Escola de Enfermagem de Ribeirão Preto da Universidade de São Paulo; 2009.

10. Bardin L. Análise de conteúdo. Lisboa: Ediçōes 70; 2010.

11. Orem ED. Nursing: concepts of practice. 6a ed. St Louis (Mo/USA): Mosby; 2001. 12. Ministério da Saúde (BR). Conselho Nacional de Saúde. Resolução 196/96. 13. OMS. Classificação de transtornos mentais e comportamento da CID-10: descrições clínicas e diretrizes diagnósticas. Porto Alegre: Artes Médicas; 1993. 14. Gonçalves SSPM, Tavares CMM. Atuação do enfermeiro na atenção ao usuário de álcool e outras drogas nos serviços extra-hospitalares. Esc Anna Nery Rev Enferm. 2007;11(4):586-92.

15. Cunha ACF, Santos TF. Autilização do grupo como recurso terapêutico no processo da terapia ocupacional com clientes com transtornos psicóticos: apontamentos bibliográficos. Cad Ter Ocupac UFSCar. 2009;17(2):133-46. 16. Lopes EM, Anjos SJSB, Pinheiro AKB. Tendências das açōes de educação em saúde realizadas por enfermeiros no Brasil. Rev Enferm UERJ. 2009;17(2):273-7. 17. Tamai $S A B$. Avaliação de um programa de promoção da saúde na qualidade de vida e no estado de bem-estar em idosos [tese]. São Paulo: Faculdade de Medicina da Universidade de São Paulo; 2010.

18. Soares LC, Santana MG, Thofehrn MB, Dias DG. Educação em Saúde na modalidade grupal: relato de experiência. Ciênc Cuid Saúde. 2009;8(1):118-23. 